Check for updates

Cite this: J. Mater. Chem. C, 2021, 9, 11180

Received 7th February 2021 Accepted 21st June 2021

DOI: $10.1039 / \mathrm{d} 1 \mathrm{tc} 00604 \mathrm{e}$

rsc.li/materials-c

\title{
A switchable multimode microlaser based on an AIE microsphere $\dagger$
}

\author{
Fengyan Song, ${ }^{a}$ Chunhuan Zhang, ${ }^{\mathrm{b}}$ Haiyun Dong, ${ }^{\mathrm{b}}$ Yuqin Fan, ${ }^{\mathrm{b}}$ Ming-Yu Wu, ${ }^{\mathrm{c}}$ \\ Guogang Shan, (D) ${ }^{d}$ Puxiang Lai, (D) ${ }^{e}$ Hui Gao, (D)*ac Yong Sheng Zhao (D)*b and \\ Sijie Chen (D)*c
}

\begin{abstract}
Switchable multimode microlasers are of great significance to the development of photonic devices with high integration levels. Herein, we demonstrate an acid/alkaline gas-responsive multimode AlEgenastarch microsphere-based microlaser. The aggregation-induced emission (AIE) active fluorescent dye ASCPI was used as the gain medium in this study. ASCPI was weakly emissive in water but became highly emissive when introduced to a starch microsphere as a guest molecule. The resultant ASCPI@starch microsphere worked well as a typical whispering-gallery-mode microlaser. The laser mode wavelengths were sizedependent. Due to the sensitivity of ASCPI to $\mathrm{pH}$, the output of the microlaser could be switched to a shorter wavelength by acetic acid vapor treatment or a longer wavelength by $\mathrm{NH}_{3}$ vapor treatment. This work will provide useful enlightenment for the rational design of effective switchable lasers using AIE materials with simple preparation procedures.
\end{abstract}

\section{Introduction}

Miniaturized lasers have gained increasing interest owing to their applications in chemical and biological sensing, ${ }^{1-4}$ laser display, ${ }^{5-7}$ imaging, ${ }^{8}$ and on-chip optical interconnects. ${ }^{9-12}$ Wavelength-tuneable lasers with the capability of delivering intense coherent light signals across a broad spectral range open up particularly interesting opportunities for highly integrated photonic devices. In the past few decades, laser switches have already been achieved by regulating the resonant mode via approaches like tuning the size or refractive index of the microcavities. ${ }^{13}$ Besides, tailoring the gain region of lasing materials has also been developed as a promising alternative approach to switching the lasing wavelength. However, for a traditional semiconductor, its energy band structure is generally fixed, which makes it difficult to dynamically modulate its gain region to realize laser switches. In contrast, organic

\footnotetext{
${ }^{a}$ School of Aeronautic Science and Engineering, Beihang University, Beijing, 100191, P. R. China.E-mail: h.gao@buaa.edu.cn

${ }^{b}$ Key Laboratory of Photochemistry, Institute of Chemistry,

Chinese Academy of Sciences, Beijing 100190, China. E-mail: yszhao@iccas.ac.cn ${ }^{c}$ Ming Wai Lau Centre for Reparative Medicine, Karolinska Institutet, Hong Kong, China. E-mail: sijie.chen@ki.se

${ }^{d}$ Institute of Functional Material Chemistry, Faculty of Chemistry, Northeast Normal University, Renmin Road 5268, Changchun 130024, P. R. China ${ }^{e}$ Department of Biomedical Engineering, The Hong Kong Polytechnic University, Hong Kong, China

$\dagger$ Electronic supplementary information (ESI) available. See DOI: 10.1039/ d1 tc00604e
}

materials with abundant energy levels and tailorable excitedstate processes show great potential for tailoring the gain region, and thus enabling the fabrication of wavelengthswitchable microlasers. ${ }^{14-19}$ Facile fabrication and excellent compatibility of organic materials are also advantageous compared with those of conventional semiconducting materials. To date, wavelength-tunable lasers based on organic materials can be realized by modulating the energy level of an excited state $^{20-24}$ or mode variation of the optical resonator. ${ }^{15,25-27}$ The excited-state energy levels of organic gain materials are sensitive to surrounding environments, thereby enabling the development of feasible methods for controllably switching the lasing wavelength via tuning the external stimuli. Previous reports have demonstrated that laser wavelength could be tuned by external stimuli, including humidity, ${ }^{28}$ temperature, ${ }^{23,29}$ light, ${ }^{30}$ vapor, ${ }^{20,21}$ solvent, ${ }^{31}$ etc. However, reports on gas-responsive micro-/ nanolasers are limited. ${ }^{20,21,31}$

Among organic gain materials, aggregation-induced emission luminogens (AIEgens) are a new type of luminescent materials, which are non-emissive when molecularly dissolved, but induced to luminesce by aggregate formation. ${ }^{32-38}$ AIEgens used as optical gain materials of microlasers can take advantage of the inherent AIE characteristics to improve lasing performance, including drastic threshold reduction and favorable lasing stability. ${ }^{20,39-41}$ In addition, these AIEgen-based miniaturized lasers can improve their laser performance with much higher doping concentrations. ${ }^{39}$ However, there are only a few reports of AIEgen-based miniaturized lasers. ${ }^{39,42}$ Reports of the environment-responsive emission behavior of AIEgen-based 
miniaturized lasers are more limited in the literature. The excitedstate intramolecular charge transfer (ICT) ${ }^{43-46}$ effect endows AIEgens with high sensitivity to the microenvironment. Therefore, AIEgens with ICT effect may be ideal candidates to fabricate robust wavelength-switchable microlasers.

Herein, we report a switchable multimode microlaser based on the ASCPI@starch microsphere. The cationic AIEgen, ASCPI, was originally weakly emissive in water solution due to its AIE and ICT properties. When mixed with starch granules, ASCPI could readily bind to the starch. The host-guest interaction restricted the intramolecular motions of the AIE molecules and strong fluorescence was triggered with blue-shifted emission color. An acidic gas could protonate ASCPI, which would consequently weaken the electron density of the donor system and therefore lead to a blue-shift of the gain region. In contrast, an alkaline gas $\left(\mathrm{NH}_{3}\right)$ provided a more polar environment for these ICT chromophores and thus led to a red-shifted gain behavior. As a result, we discovered a method of tuning lasing wavelength in the starch microparticles based on the acid/baseresponsive AIEgen. These results offer a novel understanding of the stimulated emission of AIEgens and provide useful enlightenment for the rational design of miniaturized lasers with desired performance.

\section{Experimental section}

\section{AIEgen preparation}

AIEgen (ASCPI) was synthesized according to the previous literature. ${ }^{47}$

\section{Extraction process of potato starch granules}

Potato starch granules were extracted from a fresh potato purchased at a local market. The potato was sliced into flakes, soaked in pure water, and vigorously shaken for 30-60 min. Potato starch granules were collected by centrifuging the solution (4000 rpm for $5 \mathrm{~min}$ ), followed by further washing and purification through three centrifugation cycles with pure water. Finally, the as-prepared fresh potato starch granules were collected and dried in an oven at $40{ }^{\circ} \mathrm{C}$.

\section{Preparation of ASCPI@starch}

$0.4 \mathrm{mg}$ of starch granules were immersed in $1 \mathrm{~mL}$ of pure water and sonicated for $5 \mathrm{~s}$. Then $10 \mu \mathrm{L}$ of ASCPI $\left(10^{-3} \mathrm{M}\right.$ in DMSO $)$ was dropped into the $\operatorname{starch} / \mathrm{H}_{2} \mathrm{O}$ solution, shaken by hand and left at ambient temperature for $24 \mathrm{~h}$. The mixture was centrifuged and washed with pure water several times until no characteristic emission was observed in the supernatant upon excitation, and then dried in an oven at $40{ }^{\circ} \mathrm{C}$.

\section{Measurements}

The absorption and fluorescence spectra were measured with a PerkinElmer Lambda-950 spectrophotometer and an Edinburgh FLS-1000 instrument, respectively. The time-resolved photoluminescence (TRPL) was measured with an Edinburgh FLS-1000 spectrofluorometer system equipped with an EPLED-360.
The absolute luminescence quantum yield was measured on an Edinburgh FLS 980 fluorescence spectrophotometer equipped with an integrating sphere $(0.1 \mathrm{~nm}$ step size, 0.3 second integration time, and 5 repeats). The morphology of the microsphere was examined with scanning electron microscopy (SEM, FEI Nova NanoSEM450). X-ray diffraction measurements were conducted on a D/max-2550 PC X-ray diffractometer with $\mathrm{Cu} \mathrm{K} \alpha$ radiation. Bright-field optical images and fluorescence microscopy images were taken using an inverted fluorescence microscope (Nikon Ti-U), by exciting the samples with a mercury lamp. Lasing measurements were performed on a home-built far-field microphotoluminescence system. The microsphere was locally excited with a focused $400 \mathrm{~nm}$ femtosecond laser (fs-laser), which was generated from the second harmonic of the fundamental output of a regenerative amplifier (Solstice, Spectra-Physics, $800 \mathrm{~nm}$, $100 \mathrm{fs}$, and $1 \mathrm{kHz}$ ).

\section{Results and discussion}

A typical AIEgen sensitive to $\mathrm{pH}$ was chosen as a model molecule to demonstrate the concept. Fig. 1a shows the chemical structure of the AIEgen, 4-(4-(1-cyano-2-(4-(dimethylamino)phenyl)vinyl)phenyl)-1-methylpyridin-1-ium iodide (ASCPI). ASCPI was synthesized according to a previous method. ${ }^{47}$ As illustrated in Fig. $1 \mathrm{~b}$, a typical AIE property was observed. Its dilute solution in DMSO exhibited weak photoluminescence (PL) with an emission peak at $674 \mathrm{~nm}$. Upon addition of non-polar 1,4-dioxane as a poor solvent for ASCPI into the DMSO solution with a fraction of $90 \mathrm{vol} \%$, bright red PL with a emission maximum at $650 \mathrm{~nm}$ was observed. In addition, the $\alpha_{\text {AIE }}$ value of ASCPI (Fig. 1c) was calculated to be 9.3 when $f_{\mathrm{d}}$ (1,4-dioxane fraction) was equal to $90 \%$, suggesting a typical AIE feature for this molecule. Moreover, ASCPI demonstrated a large Stokes shift $(\Delta \nu=192 \mathrm{~nm})$, which can be attributed to an excited-state ICT between the electron donor and the electron acceptor. The ICT effect was also confirmed by its solvatochromic effects ${ }^{44,45}$ (Fig. S1, ESI $\dagger$ ). ASCPI presented varied emission spectra in different solvents with wavelength changing from $666 \mathrm{~nm}$ to $688 \mathrm{~nm}$, indicating a typical charge transfer characteristic in the excited state (Fig. S1b, ESI $\dagger$ ).

Starch granules usually have spherical, ellipsoidal, or polygonal shapes, ${ }^{48,49}$ which can function as whispering gallery mode (WGM) resonators providing optical feedback for laser oscillations..$^{25,50-53}$ In this study, we explored the possibility of fabricating an AIEgen-based microlaser using starch granules as the WGM resonators. The synthetic procedure of ASCPI@starch microparticles is illustrated in Fig. 1d. Starch granules were sonicated in water. ASCPI was then added into the starch/ $\mathrm{H}_{2} \mathrm{O}$ solution, shaken by hand and left at ambient temperature for $24 \mathrm{~h}$. The obtained ASCPI@starch particles were centrifuged and washed with pure water. The doping concentration of ASCPI was $1.1 \mathrm{wt} \%$ in the starch microparticles. As shown in the bright-field image (Fig. 1e), micro-sized ASCPI@starch particles with an ellipsoidal shape could be obtained. ASCPI was weakly emissive in water (Fig. S2, ESI $\dagger$ ). This was possibly attributed to both its AIE activity and ICT property. Since this 
<smiles></smiles>

d
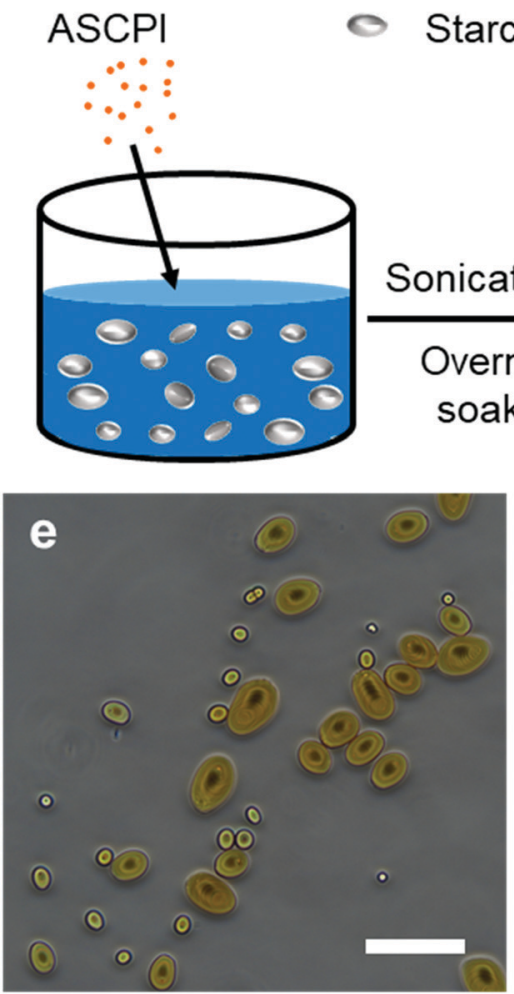
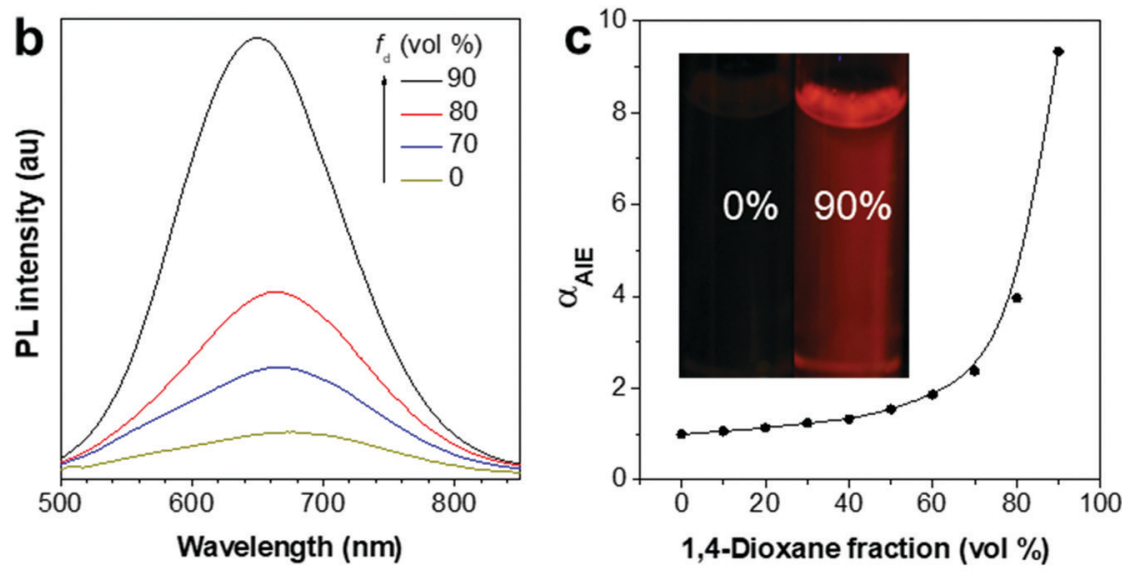

Starch Microparticles
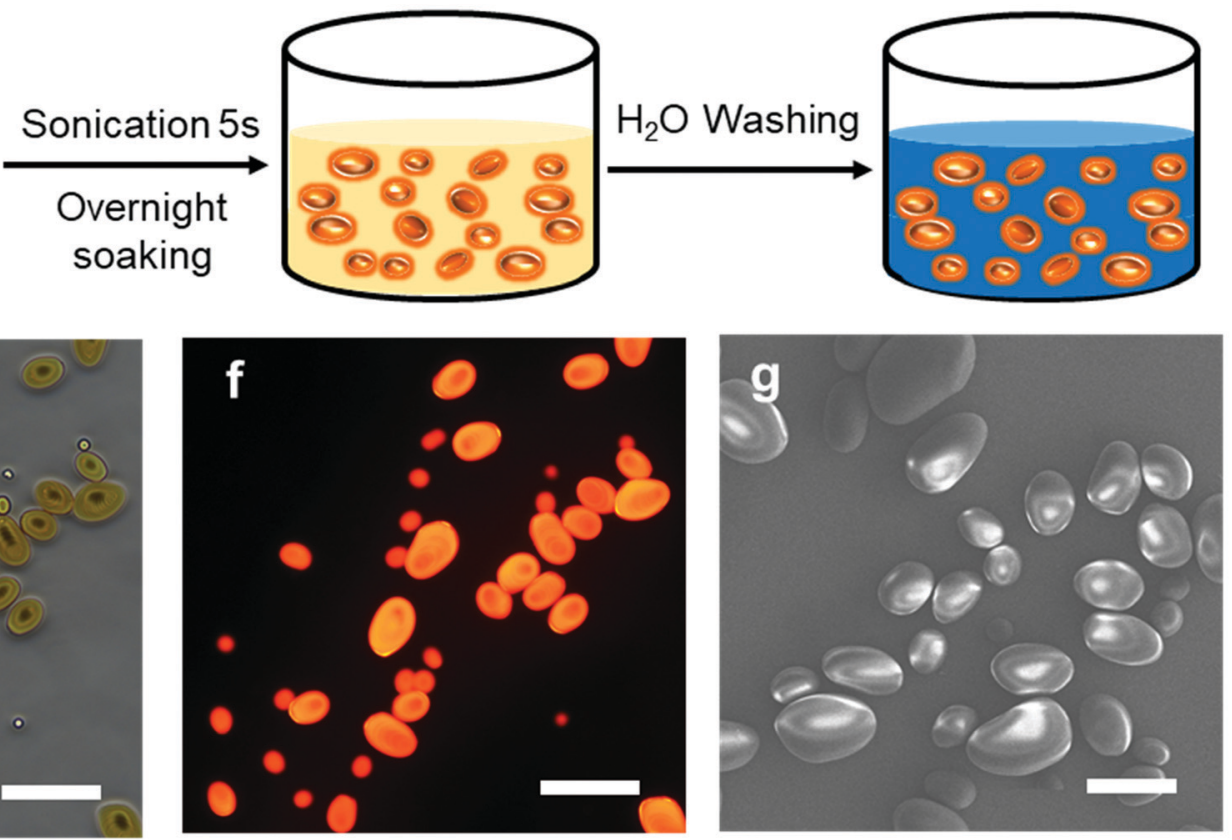

Fig. 1 (a) Molecular structure of ASCPI. (b) PL spectra of ASCPI in DMSO/1,4-dioxane mixtures with different 1,4-dioxane fractions $\left(f_{d}=0,70 \%, 80 \%\right.$, and $90 \%$ ). (c) Plot of relative emission peak intensity of ASCPI versus $f_{d}$ of DMSO/1,4-dioxane mixtures, where $\alpha_{\mathrm{AIE}}=1 / \mathrm{I}_{0}, I=$ emission intensity and $I_{0}=$ emission intensity in DMSO solution. Inset: fluorescence images of ASCPI in DMSO/1,4-dioxane mixtures with different $f_{\mathrm{d}}$ taken under $365 \mathrm{~nm}$ UV irradiation from a hand-held UV lamp at room temperature. Conditions: solution concentration: $10^{-5} \mathrm{M}$; excitation wavelength: $440 \mathrm{~nm}$. (d) Illustration of the fabrication of ASCPIastarch microparticles. (e) Bright-field microscopy image, (f) fluorescence microscopy image, and (g) SEM image of the ASCPI@starch microparticles. Scale bar: $50 \mu \mathrm{m}$.

probe carried a positive charge, it was loosely packed in water. The relatively active intramolecular motions weakened its emission. Moreover, due to its ICT property, its emission was further quenched by water. Therefore, its emission in water was rather weak. When interacting with starch granules, the dye bound to the starch and the strong host-guest interaction between the starch and dye not only restricted the intramolecular motions of the AIEgen but also changed its microenvironment. Therefore, the fluorescence of ASCPI in the starch granules was turned on (Fig. S2, ESI $\dagger$ ). As shown in Fig. 1f, bright orange-red fluorescence of the particles could be observed under UV excitation, revealing that the ASCPI molecules were successfully encapsulated by starch granules. The scanning electron microscopy (SEM) image (Fig. 1g) showed that these ASCPI@starch microparticles possessed perfect ellipsoidal shapes and ultra-smooth surfaces, possibly triggering WGM lasing at a low threshold.

We then tested the optically pumped laser behaviours of these ASCPI@starch microparticles. Here the ASCPI@starch microparticle with dimensions of $10.7 \mu \mathrm{m}$ (major axis) $\times 8.89 \mu \mathrm{m}$ (minor 

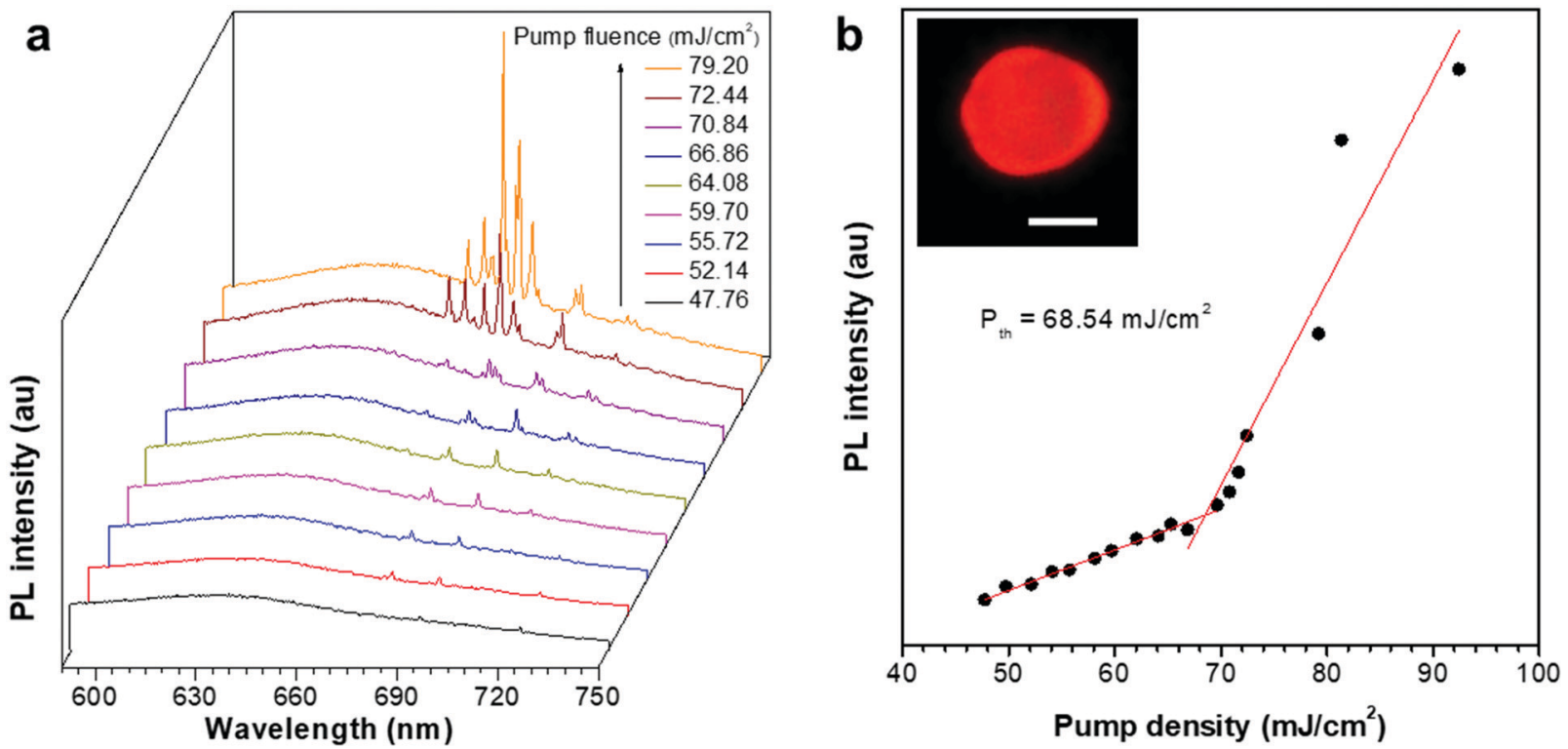

Fig. 2 (a) PL spectra recorded at the edge of an ASCPI (astarch microparticle as a function of pump density. (b) Plot of PL peak intensity as a function of pump density. Inset: fluorescence image of the ASCPIastarch microparticle (major axis $10.7 \mu \mathrm{m} \times$ minor axis $8.89 \mu \mathrm{m}$ ) under $400 \mathrm{~nm}$ pulse laser excitation. Scale bar is $10 \mu \mathrm{m}$.

axis) was excited by a femtosecond pulsed laser beam $(\lambda=400 \mathrm{~nm}$, and $200 \mathrm{fs}$ ) with increasing fluence. At low pump fluence, the ASCPI@starch microparticle exhibited a broad emission, which was peaked at about $636 \mathrm{~nm}$, corresponding to spontaneous emission (Fig. 2a). At higher pump fluence, the emission spectrum of the ASCPI@starch microparticle exhibited a set of sharp
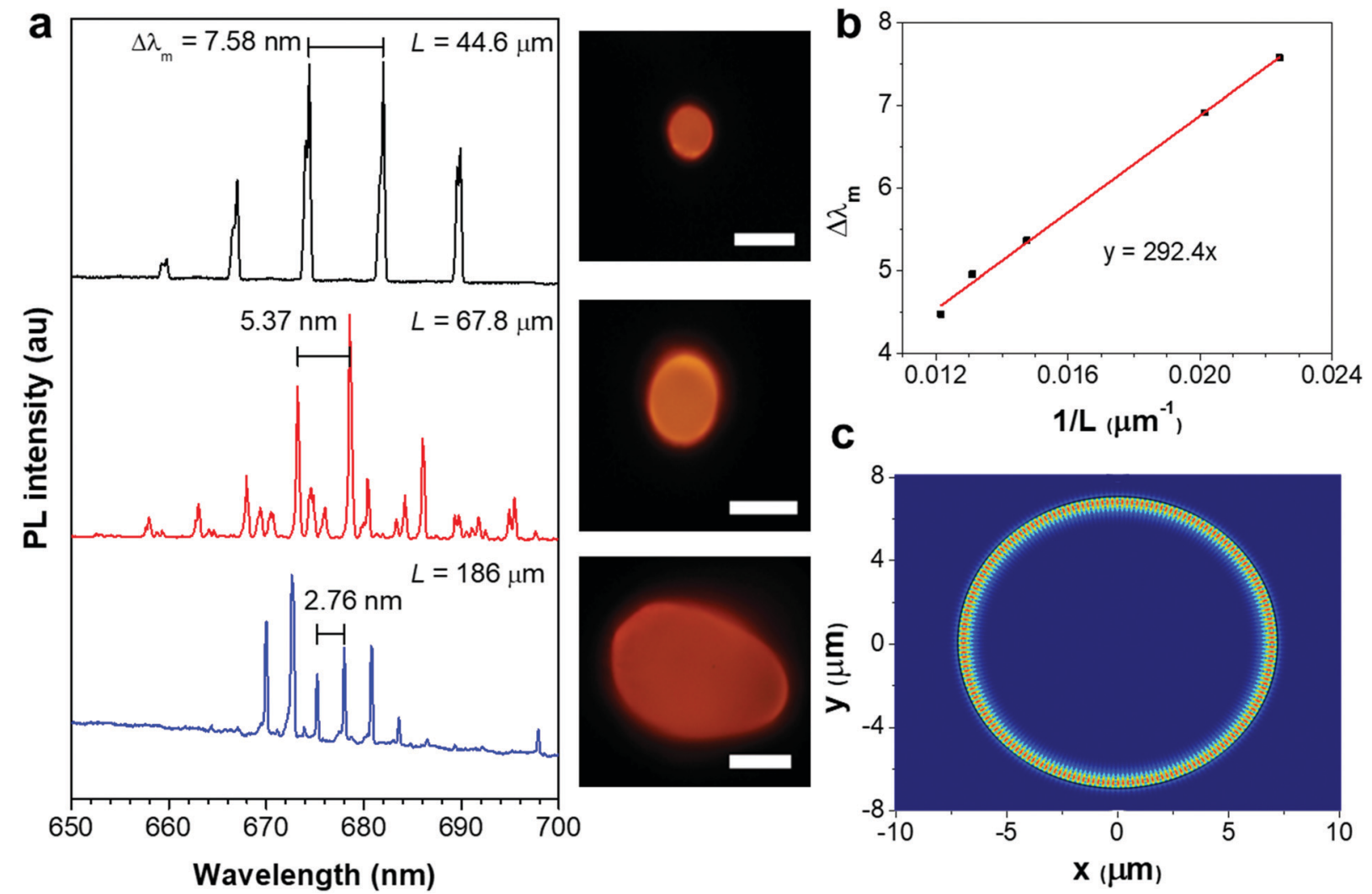

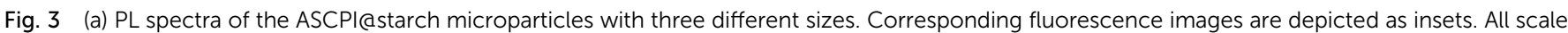

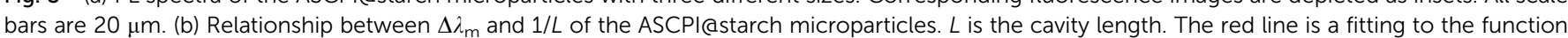
$\Delta \lambda_{\mathrm{m}}=\lambda^{2} / \mathrm{n}_{\mathrm{g}} L$, where $\lambda=682 \mathrm{~nm}$. (c) Simulated field intensity distribution $(\lambda, 682 \mathrm{~nm})$ in an ASCPI@starch microparticle with length of $44.6 \mu \mathrm{m}$. 
peaks at about $680 \mathrm{~nm}$ accompanied by a dramatically enhanced intensity, which indicated stimulated emission. The corresponding PL peak intensity versus pump fluence (Fig. 2b) revealed a clear threshold behaviour at about $68.5 \mathrm{~mJ} \mathrm{~cm}^{-2}$, which further confirmed the lasing action in the microparticles. Above the lasing threshold, the full width at half-maximum (FWHM) at $682 \mathrm{~nm}$ dramatically narrowed down to $\sim 0.4 \mathrm{~nm}$, revealing a sharp increase of temporal coherence. We also recorded the lasing intensities of the ASCPI@starch microlasers with the excitation fluence of $1.2 P_{\text {th }}$ to evaluate the stability of the prepared microlasers. The ASCPI@starch microparticles were continuously illuminated with a pulsed laser beam excitation (duration time, $100 \mathrm{fs}$; repetition rate, $1 \mathrm{kHz}$ ). As shown in Fig. S3 (ESI $\dagger$ ), sustained operation of the microlaser was achieved after scanning
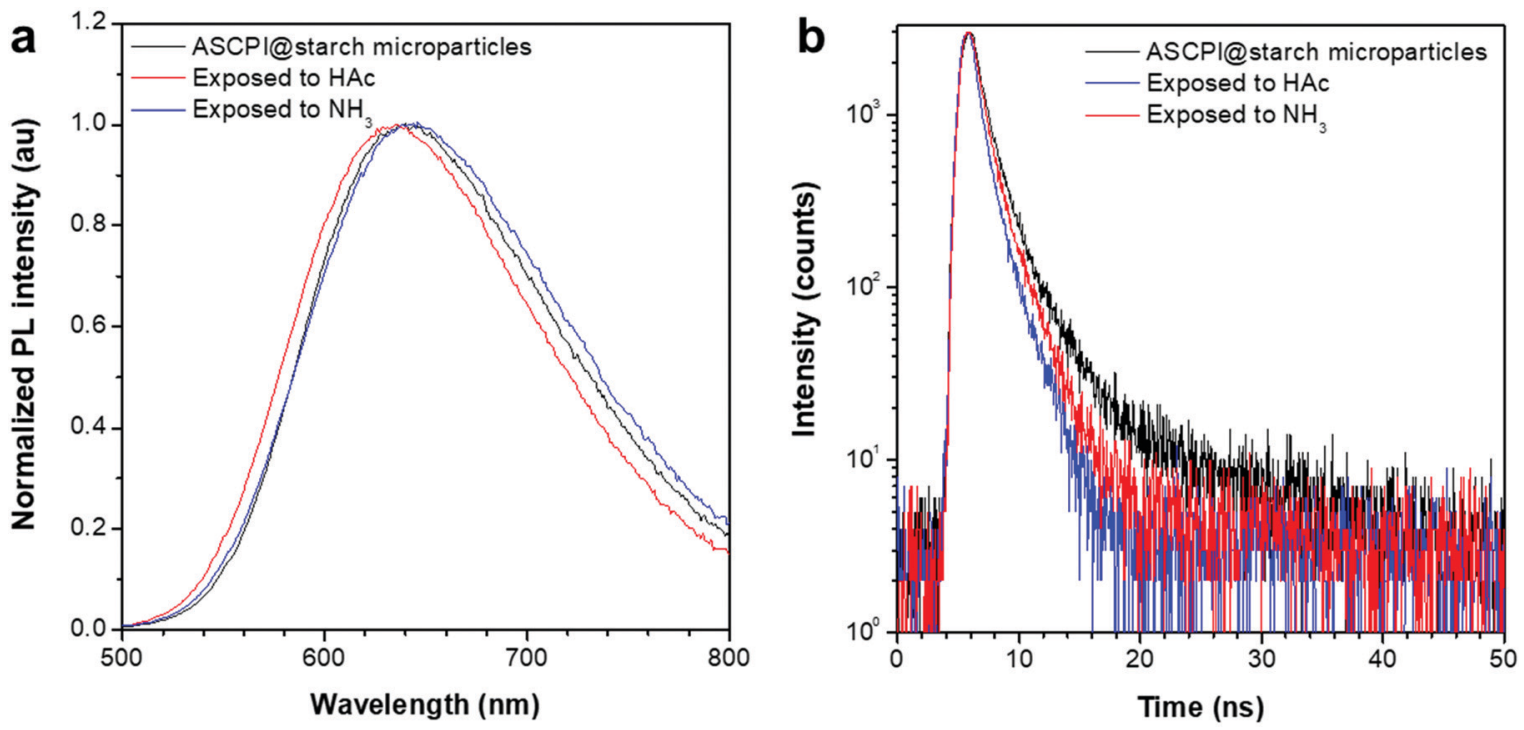

C
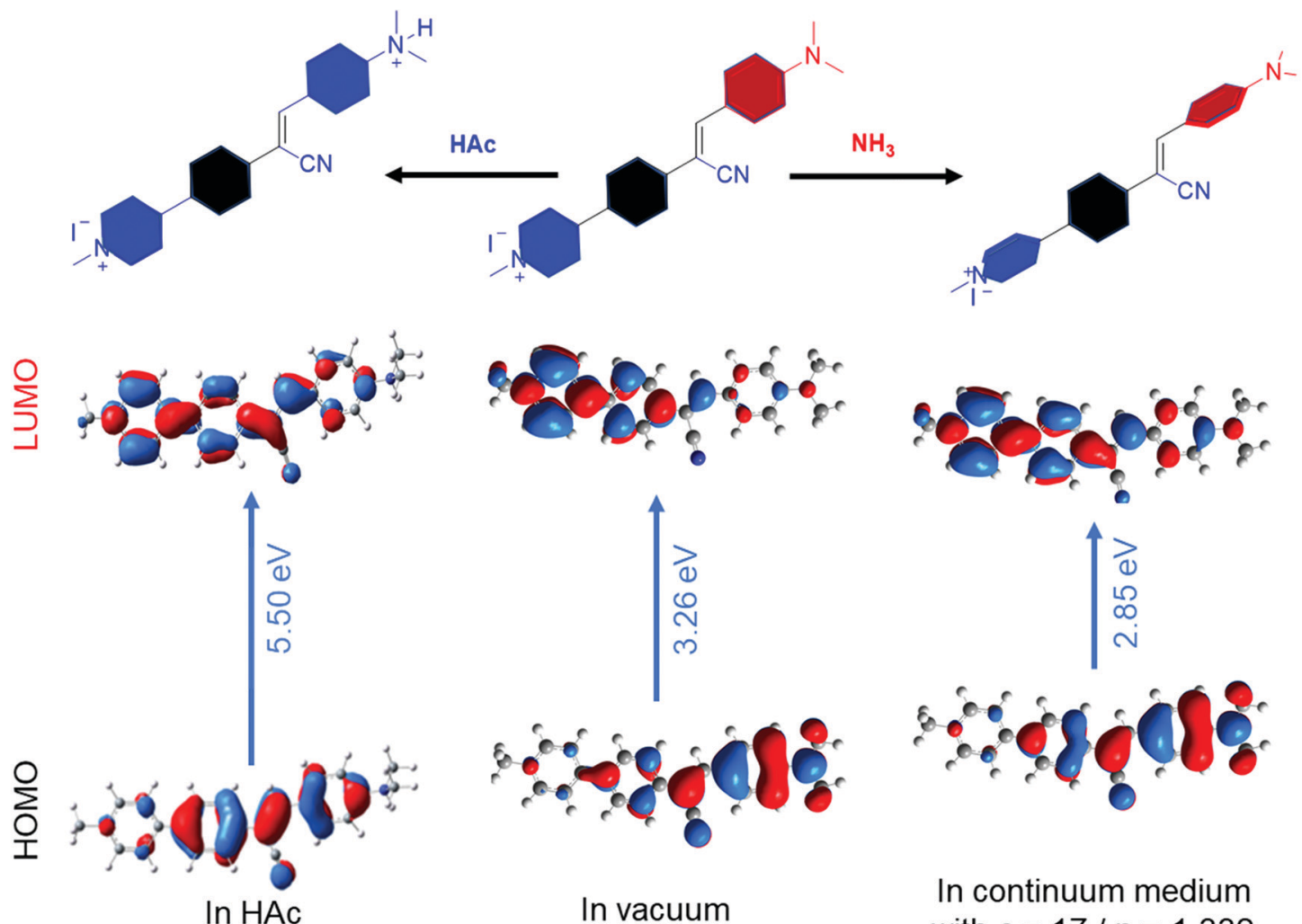

In continuum medium with $\varepsilon=17 / \mathrm{n}=1.332$

Fig. 4 (a) PL spectra of the ASCPI@starch microparticles, and ASCPI@starch microparticles in $\mathrm{HAc}$ and in $\mathrm{NH}_{3}$ atmospheres. (b) PL decay profiles of the ASCPI@starch microparticles without/with exposure to $\mathrm{HAC}$ and $\mathrm{NH}_{3}$. (c) Pictorial representations of the HOMOs and LUMOs in the ASCPI, ASCPI- $\mathrm{HAC}$ complex and ASCPI in an $\mathrm{NH}_{3}$ atmosphere. 
7000 times, accompanied by a moderate decrease in lasing intensity. This reduction of lasing intensity with increasing scanning times could be ascribed to the bleaching of dye molecules. $^{54,55}$

The lasing spectra of the ASCPI@starch microparticles with different diameters were further studied to investigate the microcavity effects. Fig. 3a illustrates the PL spectra and the corresponding images of the three ASCPI@starch microparticles with different dimensions. The mode spacing $\left(\Delta \lambda_{\mathrm{m}}\right)$ between two adjacent lasing modes decreased as the microparticle circumference $(L)$ increased from 44.6 to $186 \mu \mathrm{m}$ (Fig. 3a). For WGM resonance, $\Delta \lambda_{\mathrm{m}}$ and $L$ satisfied the equation of $\Delta \lambda_{\mathrm{m}}=\lambda^{2} / n_{\mathrm{g}} L,{ }^{25}$ where $\lambda$ was the wavelength of guided light, and $n_{\mathrm{g}}$ was the group refractive index. As shown in Fig. $3 \mathrm{~b}$, the plot of $\Delta \lambda_{\mathrm{m}}$ and $1 / L$ showed a clear linear relationship. By fitting the plot, the group refractive index $n_{\mathrm{g}}$ was identified with a value of $\sim 1.58$ at $\lambda=682 \mathrm{~nm}$ (Fig. 3b), which was consistent with the intrinsic refractive index of the starch polymer (1.53). ${ }^{56}$ These microcavity effect results verified the WGM-type cavity resonance in the ASCPI@starch microparticles. The simulated electric field intensity distribution shown in Fig. 3c further confirmed the existence of WGM in the microparticles.

As the lasing wavelength is related to the energy gap of the optical gain material, it is possible to control the gain region by tuning the energy levels of AIEgens. The ASCPI@starch microparticle exhibited a broad emission with the wavelength peak at $638 \mathrm{~nm}$ (Fig. 4a). After long-time exposure to HAc, the fluorescence peak of the ASCPI@starch microparticle exhibited a blue-shift from 638 to $633 \mathrm{~nm}$ along with a decrease of photoluminescence quantum yield (PLQY) from $14.7 \%$ to $10.6 \%$. In addition, the photoluminescence of the ASCPI@starch microparticle showed a bathochromic shift of the maximum emission wavelength from 638 to $646 \mathrm{~nm}$ (Fig. 4a) and the PLQY decreased from $14.7 \%$ to $7.6 \%$ in an $\mathrm{NH}_{3}$ atmosphere. This suggested that the external acid/alkaline gas in the atmosphere could tune the energy levels of AIEgens in the ASCPI@starch microparticles. In addition, the fluorescence lifetime of ASCPI@starch microparticles was also investigated to elucidate the inherent excited state property. As shown in Fig. 4b, the fluorescence lifetime of ASCPI@starch microparticles was $1.41 \mathrm{~ns}$. Upon exposure to $\mathrm{HAc}$ and $\mathrm{NH}_{3}$, however, the fluorescence lifetimes of the ASCPI@starch microparticles decreased to 1.03 and $1.30 \mathrm{~ns}$, respectively. According to the equation $k_{\mathrm{nr}}=(1-\varphi) / \tau$, where $\varphi$ was the PLQY, $k_{\mathrm{nr}}$ was the non-radiative decay rate constant, and $\tau$ was the fluorescence lifetime, ${ }^{57}$ the non-radiative decay rate constant $\left(k_{\mathrm{nr}}\right)$ of the ASCPI@s tarch microparticle increased after exposure to $\mathrm{HAc}$ and $\mathrm{NH}_{3}$ (Table S1, ESI $\dagger$ ). This revealed that the intramolecular rotational motions of AIEgens non-radiatively dissipated the excited-state energy in loose packings of microparticles in a HAc or $\mathrm{NH}_{3}$ atmosphere. To gain more insights into the photophysical mechanism of wavelength switching in the HAc or $\mathrm{NH}_{3}$ atmosphere, the frontier molecular orbital distributions and energy levels of ASCPI were investigated by density functional theory (DFT) using the M062X/def2vp method (Fig. 4c). ${ }^{58}$ According to the calculation results, the energy gap of protonated ASCPI was $5.50 \mathrm{eV}$ via combining with protonic acid, which was higher than that in a vacuum (3.26 eV), showing good agreement with the corresponding optical spectra after the treatment with HAc vapours. However, the energy gap of ASCPI was $2.85 \mathrm{eV}$ in the $\mathrm{NH}_{3}$ environment, which was lower than that in a vacuum $(3.26 \mathrm{eV})$ due to the ICT effect. Therefore, a red-shifted PL was observed in the optical spectra (Fig. 4a). Fourier transform infrared spectra (FTIR) of ASCPI, ASCPI + HAc and ASCPI + $\mathrm{NH}_{3} \mathrm{H}_{2} \mathrm{O}$ were also recorded. As illustrated in Fig. S4 (ESI $\dagger$ ), the FTIR transmittance spectra of the ASCPI showed a $\mathrm{C} \equiv \mathrm{N}$ stretching vibrational band at $2200 \mathrm{~cm}^{-1}$, which proved that a cyano structure was contained in the ASPCI compound. In addition, the $\mathrm{C}=\mathrm{O}$ stretching vibrational band at $1710 \mathrm{~cm}^{-1}$ was observed after treatment with acetic acid, demonstrating that a complex of ASCPI@HAc was formed. Moreover, an obvious shift of the benzene skeleton vibration from $1562 \mathrm{~cm}^{-1}$ to $1574 \mathrm{~cm}^{-1}$ was observed. This may be attributed to the conformational changes due to the ICT effect in the conjugation system after treatment with ammonia.

Inspired by the $\mathrm{HAc} / \mathrm{NH}_{3}$-responsive emission, we measured the lasing spectra in response to the variation of the HAc or $\mathrm{NH}_{3}$ atmosphere (Fig. 5a). Fig. 5b showed the lasing spectra of a typical ASCPI@starch microsphere (as shown in the dotted portion of Fig. 5c) with alternating plugs of air, $\mathrm{HAc}$ and $\mathrm{NH}_{3}$ gases. The lasing wavelength of the ASCPI@starch microsphere was tuned from about 679 to $676 \mathrm{~nm}$ when the microsphere was exposed to HAc gas (Fig. 5b). Once the ASCPI@starch microsphere was exposed to $\mathrm{NH}_{3}$ gas, the lasing wavelength was changed to about $691 \mathrm{~nm}$ (Fig. 5b). Additionally, as the HAc or $\mathrm{NH}_{3}$ vapor in the surrounding medium evaporated in air after 10 min, the PL images of the ASCPI@starch microsphere did not show obvious color change due to the weak fluorescence wavelength switching (Fig. 5c). More importantly, compared with the fluorescence wavelength switching, the microlaser used as a sensor possessed higher sensitivity to HAc and $\mathrm{NH}_{3}$ gas stimuli, which could be ascribed to the narrower FWHM in the lasing spectra (Fig. $4 \mathrm{a}$ and $5 \mathrm{~b}$ ). To further confirm the adaptability of the $\mathrm{HAc} / \mathrm{NH}_{3}$-responsive stimulated lasing properties, ASCPI@starch microspheres with different sizes were investigated under an external $\mathrm{HAc}$ or $\mathrm{NH}_{3}$ atmosphere (Fig. S5 and S6, ESI $\dagger$ ). As shown in Fig. S5 (ESI $\dagger$ ), about $3 \mathrm{~nm}$ of blueshift was observed after the exposure of the ASCPI@starch microsphere to HAc gas. On the other hand, the other sizes of the ASCPI@starch microspheres exhibited about a $7 \mathrm{~nm}$ bathochromic-shift after exposure of the microspheres to $\mathrm{NH}_{3}$ gas (Fig. S6, ESI $\dagger$ ). Overall, stimulated laser emission responsive to $\mathrm{HAc} / \mathrm{NH}_{3}$ gas could be observed in the AIEgen@starch microspheres.

To eliminate the influence of the starch resonator in these switching processes, the X-ray diffraction (XRD) patterns of the ASCPI@starch microspheres treated with acetic acid or ammonia were also investigated, as shown in Fig. S7 (ESI $\dagger$ ). The XRD pattern of the ASCPI@starch microparticles contained a series of peaks at 5.86, 17.2, 22.5 and $24.0^{\circ}$, which was in agreement with those of A-type starches. ${ }^{59}$ It was also found that the diffraction peaks at $2 \theta=17.2,22.5$ and $24.0^{\circ}$ of the ASCPI@starch 

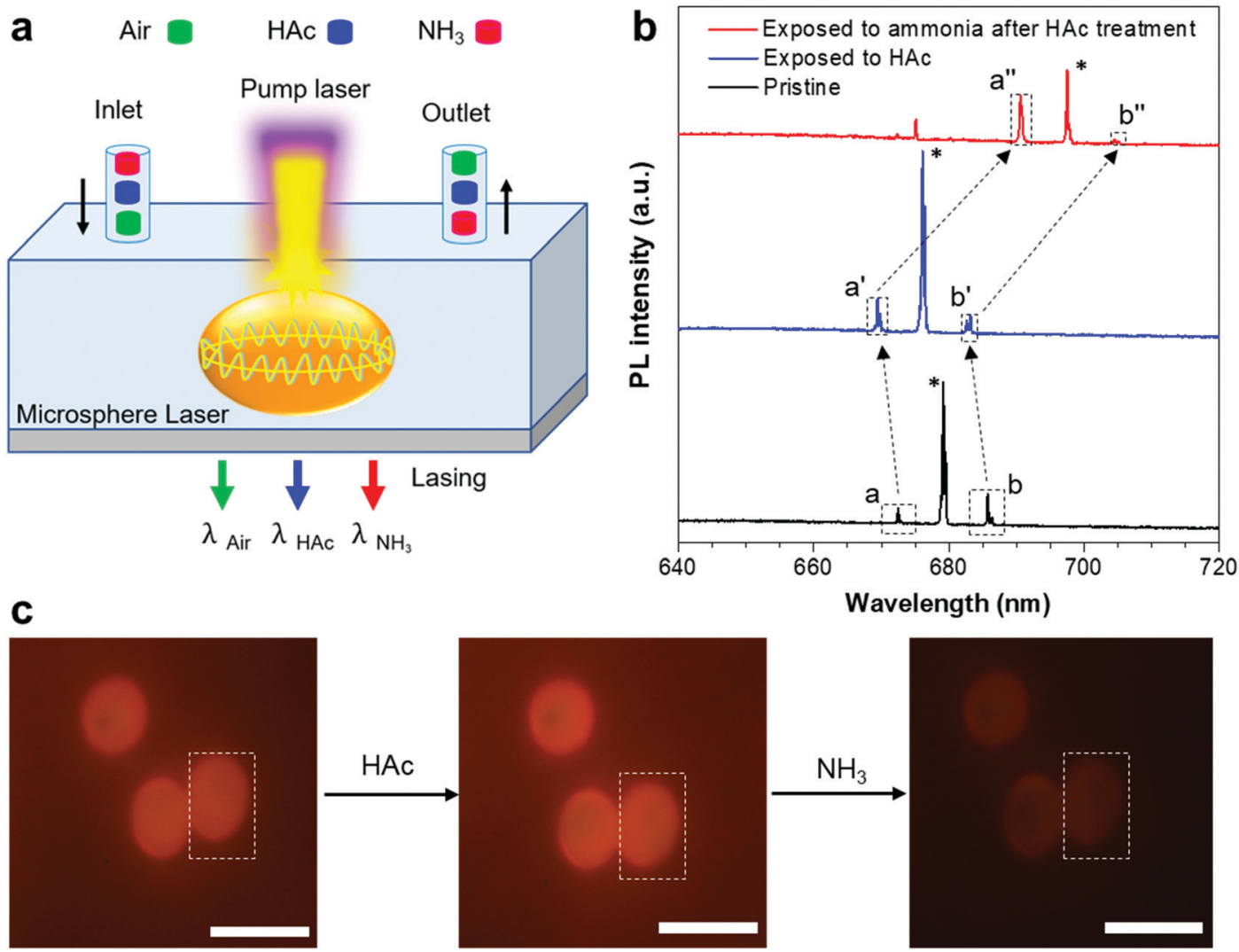

Fig. 5 (a) Schematic diagram of the ASCPIastarch microsphere laser measurement under exposure to different atmospheres. (b) Wavelength shift of lasing modes in the ASCPIastarch microsphere under alternate exposure to $\mathrm{HAc}, \mathrm{NH}_{3}$ and air, respectively. (c) Fluorescence images of an ASCPI@starch microsphere under alternate exposure to $\mathrm{HAc}_{1} \mathrm{NH}_{3}$ and air. Scale bars: $20 \mu \mathrm{m}$.

microparticles had no obvious changes after treatment with acetic acid or ammonia, suggesting that the structure of A-type starches could be maintained without influence from the presence of $\mathrm{NH}_{3}$ or HAc. In addition, the diffraction peak at $2 \theta=5.86^{\circ}$ disappeared, because the long-range ordered structure of A-type starches was destroyed during HAc treatment. These data implied that starch used as a WGM resonator had little effect on lasing wavelength switching even with treatment with acid or alkali.

\section{Conclusions}

In summary, we report an AIEgen-based switchable microlaser. This microlaser could be easily fabricated by mixing the AIE active dye ASCPI and starch granules. In the confined environment after binding to the host molecule, starch, the emission of the AIE dye ASCPI is greatly enhanced. We demonstrate that the resultant AIEgen@starch microspheres function as typical WGM lasers. More interestingly, manipulation of the laser emission can be achieved by $\mathrm{HAc} / \mathrm{NH}_{3}$ gas treatments. The protonic acid gas could weaken the electron density of the donor system and leads to blue-shifted laser behavior. On the other hand, alkaline gas $\left(\mathrm{NH}_{3}\right)$ provides a more polar environment for these ICT chromophores and thus leads to red-shifted lasing. This work presents a simple method for the fabrication of efficient AIEgen-based switchable microlasers. It is expected that more AIE-based microlasers and AIE-microlaser-based chemo- or bio-sensors will be developed in the near future.

\section{Author contributions}

S. Chen. Y. S. Zhao. P. Lai and H. Gao conceived the idea and designed the study. F. Song carried out SEM, XRD, UV, PL, PLQY and lifetime experiments. C. Zhang, H. Dong and Y. Fan preformed the lasing measurements. M.-Y. Wu synthesized the ASPCI compound. H. Gao carried out ASCPI@starch microsphere synthesis. G. Shan carried out the DFT calculations. S. Chen and Y. S. Zhao supervised this work. All the authors were involved in discussion of the results and manuscript preparation. F. Song, H. Gao, C. Zhang, S. Chen, and P. Lai drafted the manuscript with input and comments from all the authors.

\section{Conflicts of interest}

The authors declare no competing financial interest.

\section{Acknowledgements}

We would like to thank Dr. Chuen Kam and Dr Engui Zhao for critical reading of the manuscript. C. Zhang acknowledges the 
financial support from the National Natural Science Foundation of China (Grant No. 51903238). H. Gao gratefully acknowledges the financial support from the Fundamental Research Funds of Beihang University (Grant No. KG16007908). S. Chen and P. Lai acknowledge the support from the Innovation and Technology Commission (ITS/022/18, ITC, HK). S. Chen acknowledges the start-up funding from the Ming Wai Lau Centre for Reparative Medicine, Karolinska Institutet.

\section{Notes and references}

1 R.-M. Ma, S. Ota, Y. Li, S. Yang and X. Zhang, Nat. Nanotechnol., 2014, 9, 600-604.

2 L. He, Ş. K. Özdemir, J. Zhu, W. Kim and L. Yang, Nat. Nanotechnol., 2011, 6, 428-432.

3 M. Humar and S. Hyun Yun, Nat. Photonics, 2015, 9, 572-576.

4 G.-Q. Wei, X.-D. Wang and L.-S. Liao, Laser Photonics Rev., 2020, 14, 2000257.

5 F. Fan, S. Turkdogan, Z. Liu, D. Shelhammer and C. Z. Ning, Nat. Nanotechnol., 2015, 10, 796-803.

6 Z. Liu, L. Yin, H. Ning, Z. Yang, L. Tong and C.-Z. Ning, Nano Lett., 2013, 13, 4945-4950.

7 Z. Zhou, J. Zhao, Y. Du, K. Wang, J. Liang, Y. Yan and Y. S. Zhao, Angew. Chem., Int. Ed., 2020, 59, 11814-11818.

8 A. Khalatpour, A. K. Paulsen, C. Deimert, Z. R. Wasilewski and Q. Hu, Nat. Photonics, 2021, 15, 16-20.

9 L. Zhao, Y. Gao, M. Su, Q. Shang, Z. Liu, Q. Li, Q. Wei, M. Li, L. Fu, Y. Zhong, J. Shi, J. Chen, Y. Zhao, X. Qiu, X. Liu, N. Tang, G. Xing, X. Wang, B. Shen and Q. Zhang, ACS Nano, 2019, 13, 10085-10094.

10 Y. Mi, Y. Zhong, Q. Zhang and X. Liu, Adv. Opt. Mater., 2019, 7, 1900544.

11 X. Yang, Z. Shan, Z. Luo, X. Hu, H. Liu, Q. Liu, Y. Zhang, X. Zhang, M. Shoaib, J. Qu, X. Yi, X. Wang, X. Zhu, Y. Liu, L. Liao, X. Wang, S. Chen and A. Pan, ACS Nano, 2020, 14, 3397-3404.

12 H. Dong, C. Zhang, X. Liu, J. Yao and Y. S. Zhao, Chem. Soc. Rev., 2020, 49, 951-982.

13 A. Camposeo, P. Del Carro, L. Persano and D. Pisignano, Adv. Mater., 2012, 24, OP221-OP225.

14 H. Dong, C. Zhang and Y. S. Zhao, Adv. Opt. Mater., 2019, 7, 1900037.

15 J. Zhao, Y. Yan, C. Wei, W. Zhang, Z. Gao and Y. S. Zhao, Nano Lett., 2018, 18, 1241-1245.

16 C. Zhang, C.-L. Zou, H. Dong, Y. Yan, J. Yao and Y. S. Zhao, Sci. Adv., 2017, 3, e1700225.

17 Y. Lv, Z. Xiong, H. Dong, C. Wei, Y. Yang, A. Ren, Z. Yao, Y. Li, S. Xiang, Z. Zhang and Y. S. Zhao, Nano Lett., 2020, 20, 2020-2025.

18 C.-C. Yan, X.-D. Wang and L.-S. Liao, ACS Photonics, 2020, 7, 1355-1366.

19 J.-J. Wu, X.-D. Wang and L.-S. Liao, ACS Photonics, 2019, 6, 2590-2599.

20 Y. Lv, Z. Xiong, Z. Yao, Y. Yang, S. Xiang, Z. Zhang and Y. S. Zhao, J. Am. Chem. Soc., 2019, 141, 19959-19963.
21 Z. Gao, W. Zhang, Y. Yan, J. Yi, H. Dong, K. Wang, J. Yao and Y. S. Zhao, ACS Nano, 2018, 12, 5734-5740.

22 H. Dong, C. Zhang, X. Lin, Z. Zhou, J. Yao and Y. S. Zhao, Nano Lett., 2017, 17, 91-96.

23 H. Dong, Y. Wei, W. Zhang, C. Wei, C. Zhang, J. Yao and Y. S. Zhao, J. Am. Chem. Soc., 2016, 138, 1118-1121.

24 W. Zhang, Y. Yan, J. Gu, J. Yao and Y. S. Zhao, Angew. Chem., Int. Ed., 2015, 54, 7125-7129.

25 Y. Wei, X. Lin, C. Wei, W. Zhang, Y. Yan and Y. S. Zhao, ACS Nano, 2017, 11, 597-602.

26 P. Görrn, M. Lehnhardt, W. Kowalsky, T. Riedl and S. Wagner, Adv. Mater., 2011, 23, 869-872.

27 M. Karl, J. M. E. Glackin, M. Schubert, N. M. Kronenberg, G. A. Turnbull, I. D. W. Samuel and M. C. Gather, Nat. Commun., 2018, 9, 1525.

28 Q. Huang, H. Xu, M. Li, Z. Hou, C. Lv, X. Zhan, H. Li, H. Xia, H. Wang and H. Sun, J. Lightwave Technol., 2018, 36, 819-824.

29 L. Zhao, Y. Wang, Y. Yuan, Y. Liu, S. Liu, W. Sun, J. Yang and H. Li, Opt. Commun., 2017, 402, 181-185.

30 C. Qiao, C. Zhang, Z. Zhou, H. Dong, Y. Du, J. Yao and Y. S. Zhao, Angew. Chem., Int. Ed., 2020, 59, 15992-15996.

31 Y. Wei, H. Dong, C. Wei, W. Zhang, Y. Yan and Y. S. Zhao, Adv. Mater., 2016, 28, 7424-7429.

32 J. Luo, Z. Xie, J. W. Y. Lam, L. Cheng, H. Chen, C. Qiu, H. S. Kwok, X. Zhan, Y. Liu, D. Zhu and B. Z. Tang, Chem. Commun., 2001, 1740-1741.

33 J. Mei, N. L. C. Leung, R. T. K. Kwok, J. W. Y. Lam and B. Z. Tang, Chem. Rev., 2015, 115, 11718-11940.

34 Z. Zhao, H. Zhang, J. W. Y. Lam and B. Z. Tang, Angew. Chem., Int. Ed., 2020, 59, 9888-9907.

35 B. Liu and B. Z. Tang, Angew. Chem., Int. Ed., 2020, 59, 9788-9789.

36 H. Zhang, Z. Zhao, A. T. Turley, L. Wang, P. R. McGonigal, Y. Tu, Y. Li, Z. Wang, R. T. K. Kwok, J. W. Y. Lam and B. Z. Tang, Adv. Mater., 2020, 32, 2001457.

37 J. Yang, M. Fang and Z. Li, Aggregate, 2020, 1, 6-18.

38 F. Song, Z. Zhao, Z. Liu, J. W. Y. Lam and B. Z. Tang, J. Mater. Chem. C, 2020, 8, 3284-3301.

39 W. Liu, H. Yu, R. Hu, T. Xu, Y. Lun, J. Gan, S. Xu, Z. Yang and B. Z. Tang, Small, 2020, 16, 1907074.

40 C. Wei, S.-Y. Liu, C.-L. Zou, Y. Liu, J. Yao and Y. S. Zhao, J. Am. Chem. Soc., 2015, 137, 62-65.

41 G.-Q. Wei, Y.-C. Tao, J.-J. Wu, Z.-Z. Li, M.-P. Zhuo, X.-D. Wang and L.-S. Liao, J. Phys. Chem. Lett., 2019, 10, 679-684.

42 J. Gierschner, S. Varghese and S. Y. Park, Adv. Opt. Mater., 2016, 4, 348-364.

43 Z. Zhao, H. Su, P. Zhang, Y. Cai, R. T. K. Kwok, Y. Chen, Z. He, X. Gu, X. He, H. H. Y. Sung, I. D. Willimas, J. W. Y. Lam, Z. Zhang and B. Z. Tang, J. Mater. Chem. B, 2017, 5, 1650-1657. 44 S. Liu, X. Zhou, H. Zhang, H. Ou, J. W. Y. Lam, Y. Liu, L. Shi, D. Ding and B. Z. Tang, J. Am. Chem. Soc., 2019, 141, 5359-5368.

45 F. Song, Z. Xu, Q. Zhang, Z. Zhao, H. Zhang, W. Zhao, Z. Qiu, C. Qi, H. Zhang, H. H. Y. Sung, I. D. Williams, J. W. Y. Lam, 
Z. Zhao, A. Qin, D. Ma and B. Z. Tang, Adv. Funct. Mater., 2018, 28, 1800051.

46 Z. Zhao, C. Chen, W. Wu, F. Wang, L. Du, X. Zhang, Y. Xiong, X. He, Y. Cai, R. T. K. Kwok, J. W. Y. Lam, X. Gao, P. Sun, D. L. Phillips, D. Ding and B. Z. Tang, Nat. Commun., 2019, 10, 768.

47 C. Y. Y. Yu, W. Zhang, R. T. K. Kwok, C. W. T. Leung, J. W. Y. Lam and B. Z. Tang, J. Mater. Chem. B, 2016, 4, 2614-2619.

48 Y. Tan, K. Xu, L. Li, C. Liu, C. Song and P. Wang, ACS Appl. Mater. Interfaces, 2009, 1, 956-959.

49 X. Xu, R. G. F. Visser and L. M. Trindade, in Starch Polymers, ed. P. J. Halley and L. Avérous, Elsevier, Amsterdam, 2014, pp. 79-102.

50 J. Yang Zhao, L. Chen, J. Li, H.-H. Chen, X. Zhang and H. Zhou, IOP Conf. Ser.: Mater. Sci. Eng., 2020, 461, 012092.
51 H. H. Mai, T. T. Nguyen, K. M. Giang, X. T. Do, T. T. Nguyen, H. C. Hoang and V. D. Ta, Soft Matter, 2020, 16, 9069-9073.

52 X. Liu, S. T. Ha, Q. Zhang, M. de la Mata, C. Magen, J. Arbiol, T. C. Sum and Q. Xiong, ACS Nano, 2015, 9, 687-695.

53 V. D. Ta, R. Chen and H. D. Sun, Adv. Mater., 2012, 24, OP60-OP64.

54 Y. Fan, C. Zhang, Y. Du, C. Qiao, K. Wang, Y. Hou, J. Yao and Y. S. Zhao, Adv. Funct. Mater., 2021, 2103031.

55 D. Nilsson, S. Balslev, M. M. Gregersen and A. Kristensen, Appl. Opt., 2005, 44, 4965-4971.

56 I. Capron, P. Robert, P. Colonna, M. Brogly and V. Planchot, Carbohydr. Polym., 2007, 68, 249-259.

57 Q. Feng, Y. Li, L. Wang, C. Li, J. Wang, Y. Liu, K. Li and H. Hou, Chem. Commun., 2016, 52, 3123-3126.

58 Y. Zhao and D. G. Truhlar, Theor. Chem. Acc., 2008, 120, 215-241. 59 Y. Nishiyama, J.-l. Putaux, N. Montesanti, J.-L. Hazemann and C. Rochas, Biomacromolecules, 2010, 11, 76-87. 